\title{
Role of Pre-Embedded Associated Memory in Generation of Optical Illusions.
}

\author{
Krishanu Kumar Das* \\ HLG Hospital, Department of Medicine, Asansol, West Bengal
}

\begin{abstract}
This paper focuses on how our pre-embedded associated memories take part in the generation of optical illusions. According to this study optical illusions are more related to associated memories of brain, rather than optical factors. Associated memory means sets of memories associated with different objects and situations. For that, whenever we see a cup, we expect to see 'tea' or 'coffee' within it. Or whenever we look at the sky we anticipate to see 'stars' or 'clouds' in it. Retrieval of these associated memories may be triggered reflexively. Optical illusion takes the route to deceive the retrieval of the firmly embedded associated memory. Whenever we see an image, we cannot concentrate on every point of it. We concentrate our vision on a particular point, which is known as focal point. Our peripheral vision is partly constructed on imagination from experience and understanding of overall impression of the image. Our focal vision though challenges impossibility, our peripheral vision does not challenge impossibility, instead it lies between possibility and impossibility. In case of a firmly pre-embedded associated memory, our mind often perceives the image according to the pre-instituted memory and knowledge, rather than actual image, particularly in peripheral vision.
\end{abstract}

Keywords: Memory, Associated Memory, Visual Perception; Illusion; Optical illusions.

Accepted on April 09, 2017

\section{Introduction}

Perception is receival and interpretation of a sensory stimulation. These sensory data come both from the environment and from within the body. But perceiving is selective. At any given moment hundreds of stimuli are impinging on our sense organs; which particular one will be attended that the organism selects either consciously or subconsciously. Our conscious selection of perceiving sensory stimulation depends on needs, interests, desires, attitude and mental set up. Some theorists believe this filtering of information processing occurs at the level of sense organs or very early stages of input processing [1], while others argue that filtering takes place at the later stages of the information flow [2]. The anomalies of perception, however, irrespective of the site of filtering, could be classified into three types (1) Irregularities in perception (2) Illusions and (3) Hallucinations. An illusion is a misinterpretation of a stimulus, like seeing a rope as a snake. Whereas hallucination is experiencing a perception without any relevant stimulus, like seeing a snake although there is no stimulus. Illusion should not be called an anomaly always, because it is a normal phenomenon and appears in normal individuals. We call it an illusion because it does not agree with our other perceptions. There are various reasons, from which illusions could be generated, (a) Illusions due to lack of attention and concentration - like overlooking a misprint, interpreting the meaning of something from the general context. (b) Emotionally determined illusions - in the dark, many people experience a shadow or an indistinct object as human figure; or any insignificant sound as footsteps made by human. In these cases the mind sets itself emotionally predetermined to experience something. (c) Illusions driven by expectations or preconceived ideas - there are all categories of illusions visual, sensory, auditory, tactile, gustatory - that could be generated by expectations or driven by preconceived ideas. Optical illusions contribute to its visual sector.

\section{Now What are the Explanations Behind Optical Illusions?}

According to Gestalt psychology (a philosophy of mind of the Berlin school of experimental psychology, first introduced by Christian von Ehrenfels, 1890) "the whole is more than the sum of its parts". It partially explains our perception of complex patterns as unitary forms. Gestalt principles of visual grouping was introduced by Wertheimer (1923). Wertheimer, Kohler, and Koffka formulated many of the laws of grouping through the study of visual perception. These are (1) law of proximity (2) law of similarity (3) law of closure (4) law of symmetry (5) law of common fate (6) law of continuity (7) law of good results. As for, in the following series of circles, we see near circles as a pair (law of proximity). Some of these elements have been quantitatively incorporated in statistical inference of optimal probability or Bayesian inference in different later researches [3]. 
$\mathrm{OO}$ $\mathrm{O}$

Where according to Gestalt psychologists, perception was believed to be indirect, James J Gibson proposed the theory of 'ecological approach' to visual perception, which suggests that visual perception is the direct detection of environmental invariances, and that visual perception does not require inference or information processing [4]. That means, perceiving a tree does not consist of constructing a mental image of a tree from stimuli or light energy entering the visual system and then attributing the visual properties of the image to the tree in return, but one directly recognizes the visual characteristics of a tree [5]. Perceptual information is, thus, a direct interaction of the perceiver with the environment. Gibson's affordances are not mental structures. They are optical structures of information about the environment, and have only their objective values [6].

Unconscious inference in optical illusion was proposed by Hermann von Helmholtz. His theory states that the formation of visual impression is achieved primarily by unconscious judgments, the result of which "can never once be elevated to the plain of conscious judgements" and thus "lack the purifying and scrutinizing work of conscious thinking" [7]. According to Helmholtz's explanation of the physiology of perception, the qualities of sensations "belong only to our nervous system", and we acquire our knowledge of spatial ordering through perceiving an unchanging sequence of sense impressions of the same object. Helmholtz's theory of perception gives lead to the epistemological account of spatial properties. According to his 'sign' theory, brain's construction of phenomenon such as depth and spatial separation is learned after birth. However, this account brought him into conflict with Muller and Hering. Muller explained that the correspondence between sensation and object occurs by means of an innate configuration of sensory nerves, and Hering argued that experiencing an object in a single spatially ordered image, is inborn and not acquired.

Advancement of cognitive science affirmed that people differ in degrees and ways they process information. This processing of information, which characterizes different people is known as perceptual cognitive style. People who vary in the degrees of perception along with other aspects of their behaviour and personality may be flexible or constricted. At the flexible end, people are said to have a wider focus of attention and less dominated by internal needs and motives than the people at the constricted end [8]. Perceptual cognitive style also may be field-dependent or field-independent. A field-dependent person can unify and organize sensory inputs more effectively so that it could not be easily dissociable into its component parts, and thus emphasizes the whole over its integrating elements [9].

Cognition training is also an essential part of perceptual process. Perceptual learning, as defined by Gibson, is "an increase in the ability to extract information from the environment as a result of experience or practice with the stimulation coming from it" [10]. Gibson cites that competence of people trained in various occupations to make perceptual distinctions, cannot be exhibited by untrained people. Arnheim stated that perception and thinking are inseparable processes [11]. According to him, "the clarification of visual forms and their organization in integrated patterns as well as the attribution of such forms to suitable objects is one of the most effective training grounds of the young mind". According to Pinker, our understanding of real world is dependent on the training of mind and that also helps us to recognize shapes and forms based upon built knowledge [12-14]. Oliver Sacks showed, on study of several patients including himself with having difficulties of visual recognition, that mind can be educated through learning procedures to acquire some knowledge of codes or conventions that can help to recognize objects $[15,16]$.

\section{Visual Perception of Contour, Size, Shape, Brightness, Depth and Motion}

Visual perception is dependent on understanding of contours, shapes, brightness, colors, depth, and movement. Contours are formed whenever a marked difference occurs in the brightness or color of the background. Contours give shape to the objects in our visual field because they mark one object off from another and from background. While differences in energy levels of light across the retina are involved in the formation of most contours, the contours can sometimes be seen without any energy difference on the two sides of the contour [17]. These are so called subjective contours. In Figure 1, there is the contours of an inverted triangle even though there is no energy changes across its perceived borders except in corners. But the three angles forming the corners of the upright triangle do not produce a subjective contour.

For explanation for recognizing of size, shape, pattern of objects through visual perception, there are two fundamental approaches - 'Bottom-up' and 'Top-down' theories. Bottom-up theories take approaches that perception starts with the visual stimuli received in the eye. So they are called data-driven or stimulus driven theories. Whereas according to 'Topdown' theories, perception is driven by high-level cognitive

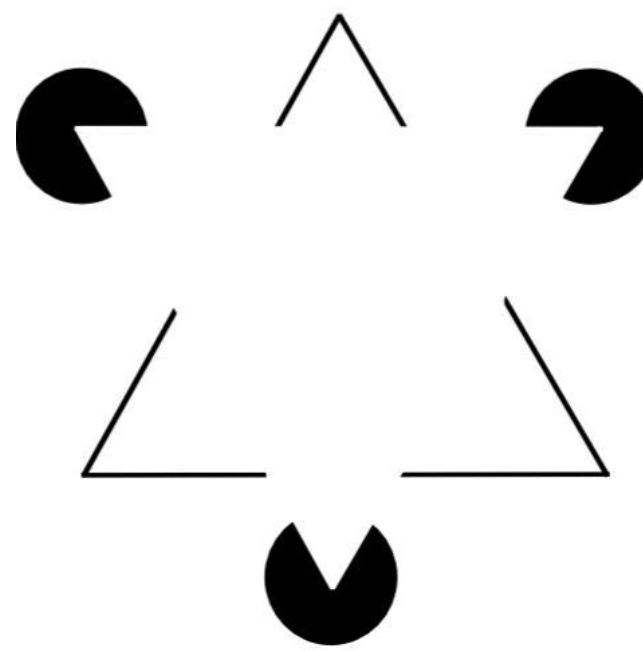

Figure 1. Kanizsa's triangle. 
processes and existing knowledge. Several other theories, proposed for recognising objects, are template, prototype and feature matching theories. According to these theories, we try to match the object with similar types of prototype, template or features stored in our memories. The features have also been distinguished between local and global features. Local features constitute small-scale detailed aspects of a given pattern, whereas global features form its overall shape. In different studies it has been shown that global information dominates over local information (global precedence effect) in visual perception $[18,19]$.

Perceptual universe is also dependent on constancies of size, shape, colour and brightness. For that, a familiar object will appear the same even in different surroundings, like coal looks black even in bright sunlight, where snow continues to look white even in darkness. Regarding constancy of size, two notable illusions could be cited, Muller-Lyer illusion (Figure 2) and Ponzo's illusion. In Muller-Lyer illusion, the straight line with arrows pointing outward seems to be longer than the straight line with arrows pointing inward. This has been explained as "misplaced" size constancy as an outside corner seems to approach in depth while an inside corner appears to recede in depth $[20,21]$.

\section{Depth}

Humans enjoy stereoscopic vision because our eyes are separated horizontally in such a degree that there occurs overlapping of images from two eyes. Images formed in retinas of two eyes are slightly different in their degrees of plane. From this difference of degrees in plane, our visual areas in brain perceive the depth of objects. More refined analysis is performed as the visual signals are passed from primary visual cortex (V1) to strata of secondary visual cortices (V2, V3, V4, and V5). But besides this 'retinal disparity', both in monocular vision and binocular vision the depth is attributed by different cues, for that we are able to get three dimensional experience from a flat painting. According to Gibson, there are sufficient contextual information in the real world to make our perceptual judgements of depth. He stated that we use these cues to judge the relative depth and
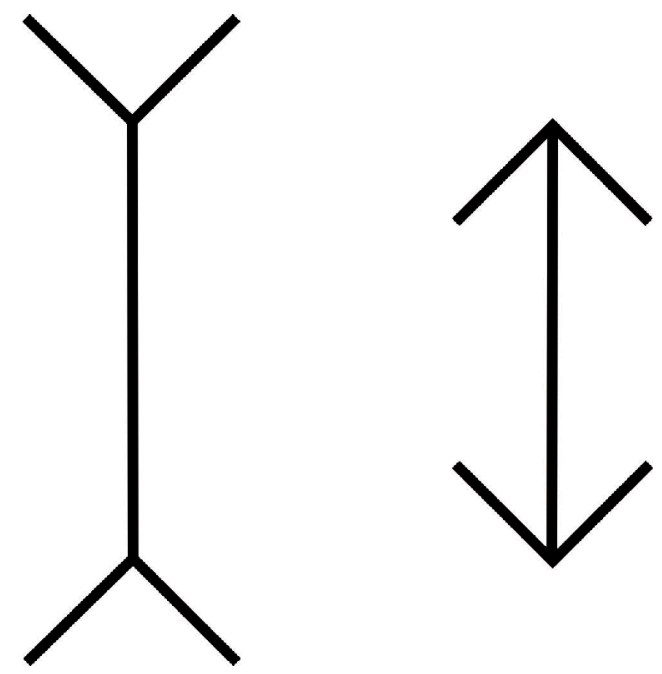

Figure 2. Muller-Lyer illusion. distance between the objects as well as between the parts of objects [22]. Biederman suggested that we recognize three dimensional form of an object by dissociating them into a number of elementary units called 'geons'. According to his recognition-by-components (RBC) theory, these geons could be arranged or rearranged in different ways to make for representation of many different objects. As these geons are view point invariant, the representation essentially does not depend on the observer's angle of seeing the object $[23,24]$.

\section{Motion}

Ability to extract motion information from the visual scene is another vital task of the visual system. Perceived motion without any energy movement across the receptor surface is called apparent motion, where the perception of the actual physical movement of objects is called real motion. How the mind can differentiate between the motion of an object and the observer's own movement, has been explained by the conceptual model of the 'brain comparator' $[20,25]$. The brain comparator is a system which compares information coming from muscle movements of both eyes and other bodily muscles with information about movements of the retinal image. When movements of muscles feed information to brain comparator, the comparator accordingly adjust for visual perception and cancels the simultaneous movements of the retinal image to understand the real motion of the object.

\section{Lateral Inhibition}

Biological approach to explain optical illusion has developed the theory based on lateral inhibition, which has been succeeded to explain the "Mach Bands Illusion", Figure 3 [26]. Here in the receptive field of the retina, the photoreceptor cells (rod cells) receiving the lighted part of the image laterally inhibit adjacent photo-receptor cells receiving the darker area of the image. As a result of which, we see increased contrast at the edges of slightly different shades. This constitutes visual acuity or sharpness of vision. However, lateral inhibition can explain some of the factors in optical illusions.

\section{Approach to Explain Optical Illusions with Methods in This Study}

According to this study the secrets behind optical illusions lie within the fact that it, somehow, deceives the associated memory of us. Association of memory refers to the set of memories regarding a particular object, person, or situation. For that, whenever we see a 'cup', we expect or predict to see 'tea' or 'coffee' within it; whenever we look at the sky,

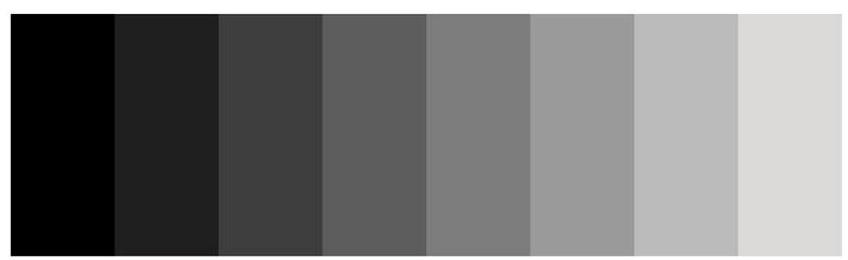

Figure 3. Mach bands illusion. 


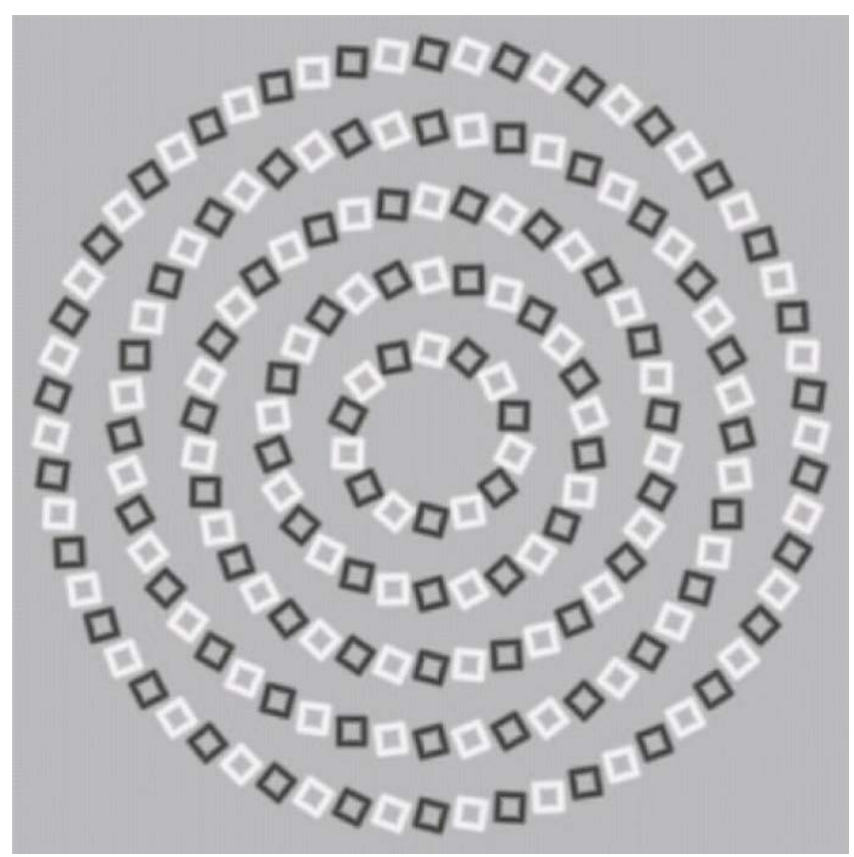

Figure 4. Intertwining illusion. The circles appear to be spiraling and intersecting.

we expect or anticipate to see 'stars' or 'clouds' in it. Because they have been embedded in individual sets of associated memories within our brain, the retrieval of which may occur reflexively. In the case of more soundly formed associated memory, we interpret the thing according to the respective set of the associated memory regarding that particular object or situation, though the real thing may not go with this preinstituted memory. And in those cases of discrepancies, optical illusions are formed.

As in Figure 4, if we look carefully to the small squares, they would seem to be down-stepping stairs. Now, if we step down, we cannot remain at the same level. So the image is contradicting our embedded and established memories - our knowledge and experience. So the illusion is forming as to be the circles of the stairs being converging.

In a simple associative learning procedure, when a cue A is presented and followed by an outcome $\mathrm{X}$, with experience, presentation of A elicits anticipation of $\mathrm{X}$. The growth of this anticipation is the process of associative learning. In other words, 'associated learning' is a learning principle based on the assumption that ideas and experiences reinforce one another and can be linked. Thus associative learning is modelled as a gradual accrual of excitatory and inhibitory connections between the stimulus units. Typically associative strength is treated as summative. So the strength of an association between stimuli stands for the entire history of learning [27].

'Associative memory' is defined as the ability to learn and remember the relationship between unrelated items [28]. Here the term 'associated memory' has been used to encompass the memories which have been related or associated through the process of associative learning.
Science of associative learning began with the pioneering studies of Pavlov and Thorndike nearly 100 years back, and continues till date as an active area of research to understand behavior and cognition in human and non-human animals. Learning and memory could not be separated. Though years of cognitive studies have laid more focus on associative processes in case of learning and on information processing in fields of memory, persistent consistencies have been observed in researches in both traditions.

Many theories, posited about basic conditioning phenomena of associative learning, are acquisition and extinction; discrimination; generalization; blocking and overshadowing; superconditioning; overexpectation; and retrospective revaluation [29-35].

Associative strength, as Rescorla and Wagner predicted in their theory of associative learning [31] can be demonstrated as following:

If associative strength of cue $\mathrm{C}$ with the outcome $\mathrm{O}$ is changed from $\mathrm{V}_{\mathrm{i}}$ to $\mathrm{V}_{\mathrm{t}}$ after trial $\mathrm{T}$, then

$$
\mathrm{V}_{\mathrm{t}}=\mathrm{V}_{\mathrm{i}}+\Delta \mathrm{V}_{\mathrm{i}, \mathrm{t}}
$$

$\Delta \mathrm{V}_{\mathrm{i}, \mathrm{t}}$ is the change of association strength and calculated by the formula -

$$
\Delta \mathrm{V}_{\mathrm{i}, \mathrm{t}}=\alpha \beta\left(\lambda-\mathrm{V}_{\mathrm{i}}\right)
$$

Here $\lambda$ is the maximum level of associative strength possible, and $\mathrm{V}_{\mathrm{i}}$ is the associative strength before trial. $\alpha$ measures the salience of the particular cue, and $\beta$ represents the salience of the particular situation, in which the outcome occurs. Rescorla and Wagner indicated $\alpha \& \beta$ as learning parameters, which are specific to particular CS (conditioned stimulus) and US (unconditioned stimulus), respectively.

Causal-model theory provides an alternative statistical concept of contingency. It states that the objective relationship between a cue and an outcome, in relation to which the accuracy of judgments is assessed, is estimated by mean of $\Delta \mathrm{P}$. This statistic $(\Delta \mathrm{P})$ is defined as the difference between the probability of the outcome given that the cue has been presented $\mathrm{P}(\mathrm{O} / \mathrm{C})$ and the probability of the outcome given that the cue has not been presented $\mathrm{P}(\mathrm{O} / \sim \mathrm{C})$.

$$
\Delta \mathrm{P}=\mathrm{P}(\mathrm{O} / \mathrm{C})-\mathrm{P}(\mathrm{O} / \sim \mathrm{C})
$$

$\sim \mathrm{C}$ signifies the absence of the cue/cause. Here it is noticeable that the concept of contingency depends not only on the proportion of cases in which the effect and cause cooccurring, but also on the proportion of cases in which the effect occurs in the absence of the possible cause. Contingency is thus distinct from the simple conditional probability of the effect on a given cause [36-39].

Our present study postulates that the development of visual perceptual inferences relies on this associative-strength depended pre-embedded memories, which is the key factor for generation of optical illusions.

Our assessment of size, shape, color, contour, consistency, 
A

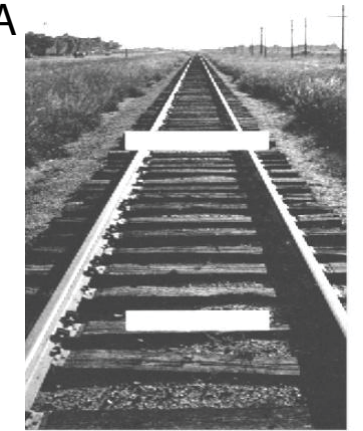

B

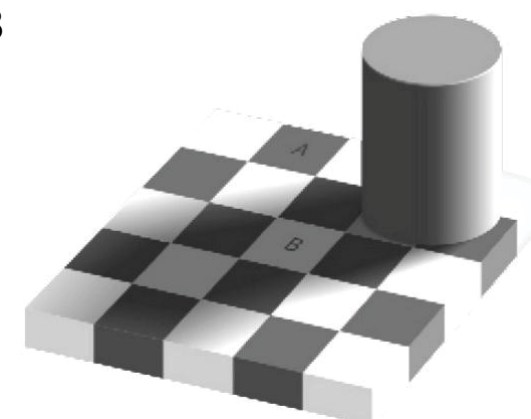

Figure 5. A) Ponzo's illusion. The white horizontal bar in the distant seems to be longer than the nearer one, though they are the same length (after Italian psychologist Mario Ponzo). B) Checker shadow illusion. The block A and the block B are of same shades of colour, but the block A appears to be darker than the block B (from Edward H Adelson).

and depth of the objects partly depends on the preabsorbed knowledge and experience about them. When we observe two objects - one far and another near, or one in light and another in shadow, our mind takes the far object as larger than what it looks, and the shadowed object brighter than what it actually appears. Let us look at Figure 5A and Figure 5B. In Figure $5 \mathrm{~A}$, two horizontal white bars are of same length, though the distant one seems to be longer. In the Figure $5 \mathrm{~B}$, the block $\mathrm{A}$ appears to be darker than the block B, though they are both of same colour. We get illusioned because, to us, it has been preconceived through our knowledge and experiences, that is through associative learning processes, that the object in distance is larger and the object in shadow is brighter than what they actually look like.

Our concept of size, shape, contour and colour partially comes from comparison with the surrounding objects, and dominancy of the overall impression of the image, which is related with our knowledge. When two objects of the same size are juxtaposed with two different sized other objects, the one in the proximity of the bigger object seems to be smaller than the one with the smaller accompanied object.
When two objects of same colour and shade are imposed on two differently shaded backgrounds, the one with the brighter background looks darker and one with the darker background looks brighter; and when they are proximated with two differently colored backgrounds, they acquire some effect of the background colour on them, like red looks differently within deep green and yellow.

Our perception of shape and contour of an object is influenced by the integrated impression or dominant background of the image. In the Figure 6A (Hering Illusion), the straight lines seem to be curved at the center. Why ? It is due to the fact that the overall impression of the image is divergence particularly at the center. When we look at the center of the image, the mind gets dominated by the idea of divergence. This background cue evokes the anticipation of outcome of divergent foreview depending on the strong association strength of the past memories. So the straight lines seem to be curved outwards near the center. In the Figure $6 \mathrm{~B}$, I have drawn a perfect circle, but because of the wavy nature of the background, which is overpowering the mind, the circle appears to be vertically oval, that is its vertical
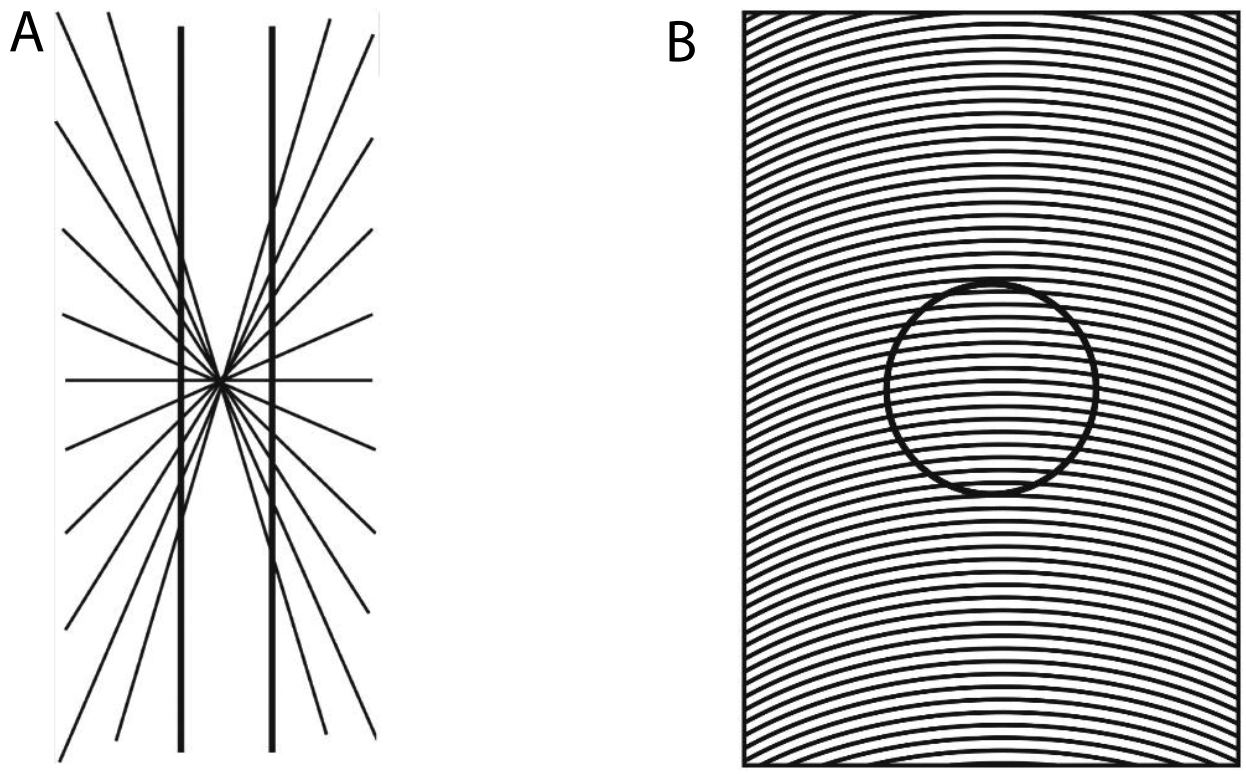

Figure 6. A) Hering illusion. Straight lines appear to be curved outwards near the center (after physiologist Ewald Hering who first described it in 1861). B) Oval circle (author). 
diameter seems to be greater than its horizontal diameter.

Through binocular vision, from the difference in angles of two images formed in two eyes, we perceive depth of objects. But besides that, we also conceive the depth, contour and three dimensional structure of any object from the knowledge of shades, shadows and contrast, which has already been constituted in our memory in course of time since the time of birth. Optical illusions take the route to betray all these firmly pre-embedded knowledge.

\section{Use of Focal Point and Peripheral Vision}

When we see an image, we cannot concentrate on every point of it. We usually concentrate our vision on a particular point or focal point of the image both in monocular and binocular vision, and perceive the periphery either through the overall interpretation of the image related with our knowledge, or through expectation or anticipation from our already formed assembled associated memories. There are two factors that mind always encounters in perception, 'the possibility' and 'the impossibility'. Our focal vision, where we concentrate our attention, challenges impossibilities; but our peripheral vision does not challenge impossibilities, rather lie between possibilities and impossibilities, if any there.

Below in Figure 7A, I have drawn an outline of a face, but in the place of eye I drew lips. When one will directly look at the lips, his mind will challenge the impossibility to get new information, new experience, and new learning. But if one concentrates on the front or lower part of the face, or do not concentrate on any point at all, only the impression of an eye will appear to him, the lips will disappear. Our peripheral view of an image is partly formed by our embedded and associated memory. Here I am deceiving embedded associated memory. In the Figure 7B, when one concentrates on the lower dots, the upper dots disappear and vice versa happens when one concentrates on the upper dots. One cannot see all of the dots at the same time. Because the dots are not expected to be there according to our existing knowledge along with overall impression of the image.

This is also a reason why peripheral vision is poorer in children than adults, because of poorer pre-embedded memories. And why pedestrian accident rate is higher in children than adults [40].

No image is formed at the optic disc (blind spot) of the retina, where the optic nerve with vessels leaves the retina. Usually in binocular vision this deficit is filled up by the image formed in the other eye because the optic disc lies $3 \mathrm{~mm}$ medial (towards the central axis of the body) to the posterior center of the retina (fovea centralis). But if we close our one eye, we do not see a hole in our vision. How the brain fills up this gap? The brain fills up this gap out of imagination from experience and overall impression of the view (Figure 8).

What we see, partly depends on what we want to see or what we anticipate to see.

\section{Moving Optical Illusion}

In case of moving optical illusions (Figure 9A \& Figure
A

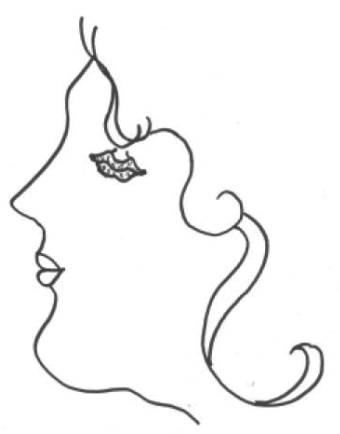

B

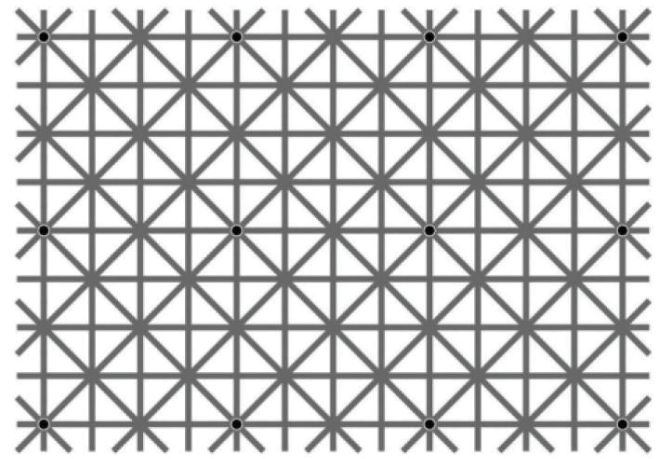

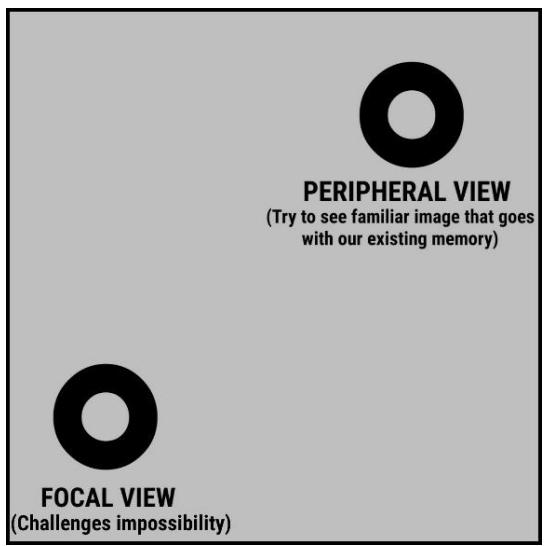

Figure 7. A) Eye-lips illusion (author). B) Disappearing dots. There are 12 black dots at the intersections of the grid. Your mind would not let you see all at a time. 
a

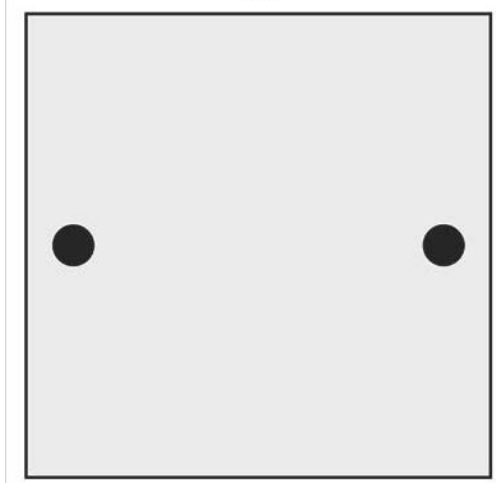

b

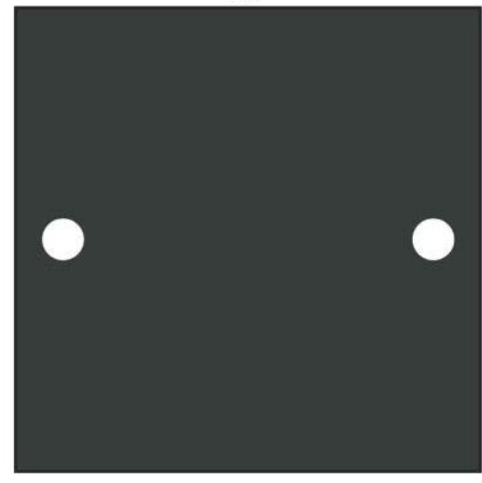

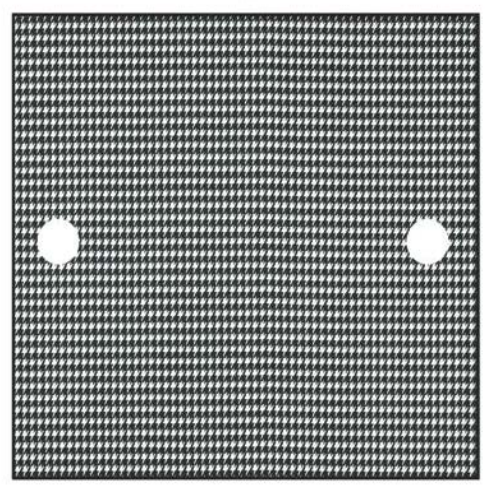

Figure 8. If we close our one eye, and fixing gaze on one small circle move forward: when the image of other circle will fall on blind spot, we will see the space according to overall impression related to our knowledge; white (a), black (b), and chequered (c).

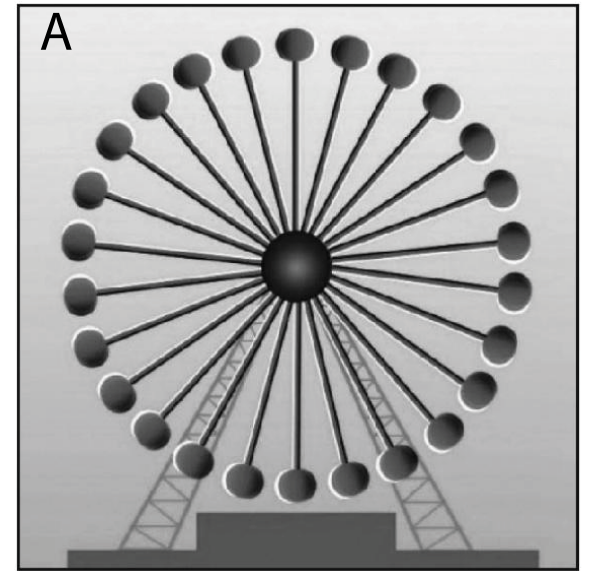

Figure 9. A) Ferris wheel illusion. The wheel seems to be rotating. B) Rotating circles: The circles seem to be rotating - inner clockwise, outer anticlockwise (author).

9B), the trick is we have to give different shades and lighted areas to the objects of the image. It is not possible when light comes from a fixed direction and objects are stationary. It is only possible when either the light source or the objects are moving. We are usually accustomed to see moving objects rather than moving light source. So the mind perceives that the objects in the image are moving. This movement is apparent motion, which occurs without the real motion of the object. This apparent motion is also dominant in peripheral vision, rather than focal vision, where mind perceives the motion out of its pre-existing knowledge.

In generating an optical illusion, whatever we do, aim would be to contradict our firmly embedded associated

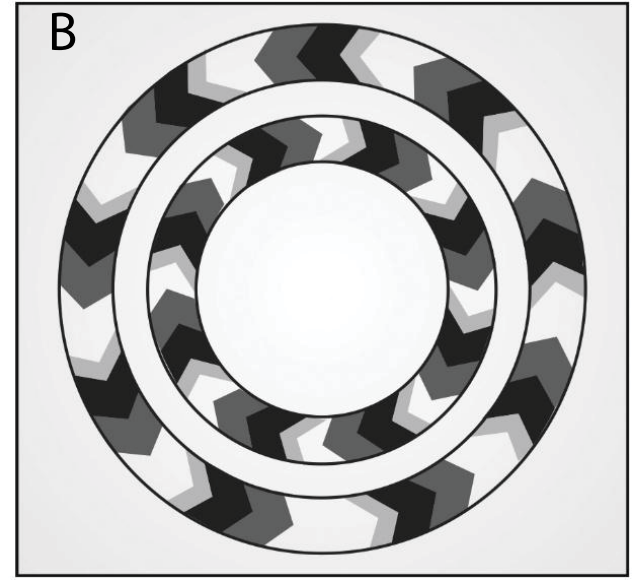

memory. Here the strength of illusion is proportional to the strength of association. If the associative strength for a given cue $i$ and outcome $j$ is $P_{i, j}$, then when the real outcome is different - the illusory strength $\mathrm{I}_{\mathrm{i}, \mathrm{j}} \propto \mathrm{P}_{\mathrm{i}, \mathrm{j}}$ (where the contextual variable is fixed).

And change of illusory strength $\Delta \mathrm{I}_{\mathrm{i}, \mathrm{j}}$ is proportional to change of associative strength $\Delta \mathrm{P}_{\mathrm{i}, \mathrm{j}}$. But it is significant to notice that when the real outcome is different, there is also simultaneous concurrent change of associative strength for a particular cue and outcome. So in the next trial both the strengths will be changed and the change of illusory strength will proportionally follow the change of associative strength, maintaining contextual variable is fixed. This predicts the 
fact that repeated exposures of an optical illusion reduce the illusory effect of the illusion to a subject.

For that, optical illusions are most successful in those cases, where association strength is high, or where in normal life lost association strength is recovered through regular experience at much higher rate.

Now, if we equate the above proportionality,

$$
\mathrm{I}_{\mathrm{i}, \mathrm{j}}=\tau \times \mathrm{C} \times \mathrm{P}_{\mathrm{i}, \mathrm{j}}
$$

Here, $\tau$ is the Illusory coefficient which is a constant, and $\mathrm{C}$ is the contextual variable. Contextual variant $\mathrm{C}$ again is the multiplication of observer's variable ' $\mathrm{O}$ ' and situational variable 'S'.

$$
\mathrm{C}=\mathrm{O} . \mathrm{S}
$$

Observer's variable 'O' depends on the observer's emotional state, mood, the amount of attention he or she is paying, and the particular area of the visual field through which the observer is receiving the illusion. Situational variable 'S' depends on litght, shades, clearness of the medium and other situational determinants at the time of the illusion to be occurring.

\section{Illusory Co-efficient}

Illusory co-efficient is not a fixed constant. It is both sense organ specific and region specific. Illusory co-efficient is different in case of different senses - visual, auditory, gustatory, olfactory, tactile, and proprioceptive.

For visual perception, the illusory co-efficient for all foveal cues of a particular colour is constant at a particular point ' $\mathrm{P}$ ' in peripheral visual field. If $\mathrm{P}$ increases the distance from fovea centralis, the illusory co-efficient also increases. As the concentration of retinal photoreceptor cells and nerve fibers are not equally distributed in all directions from fovea, $\mathrm{P}$ is both distance and direction, i.e., region specific. More clearly to say, where in retina the concentration of cells for real perception is less, the mind takes the course of imagination more from pre-established knowledge. And the illusory co-efficient for that region increases. At the blind spot the illusory co-efficient is maximum and at fovea the illusory co-efficient is minimum.
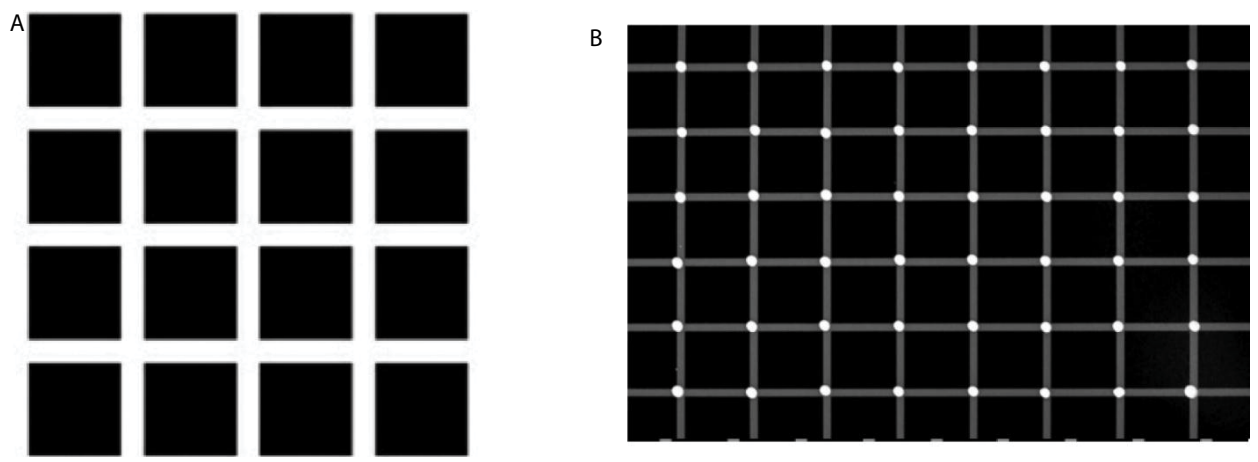

Figure 10. A) Hermann-Grid illusion. Darkness appears at the intersections of the grid which dissipates also along the borders (named after Ludimar Hermann who discovered it, 1870). B) Lingelbach illusion. A variation of H.G. illusion. Dark dots appear and disappear at the intersections (after E Lingelbach). 

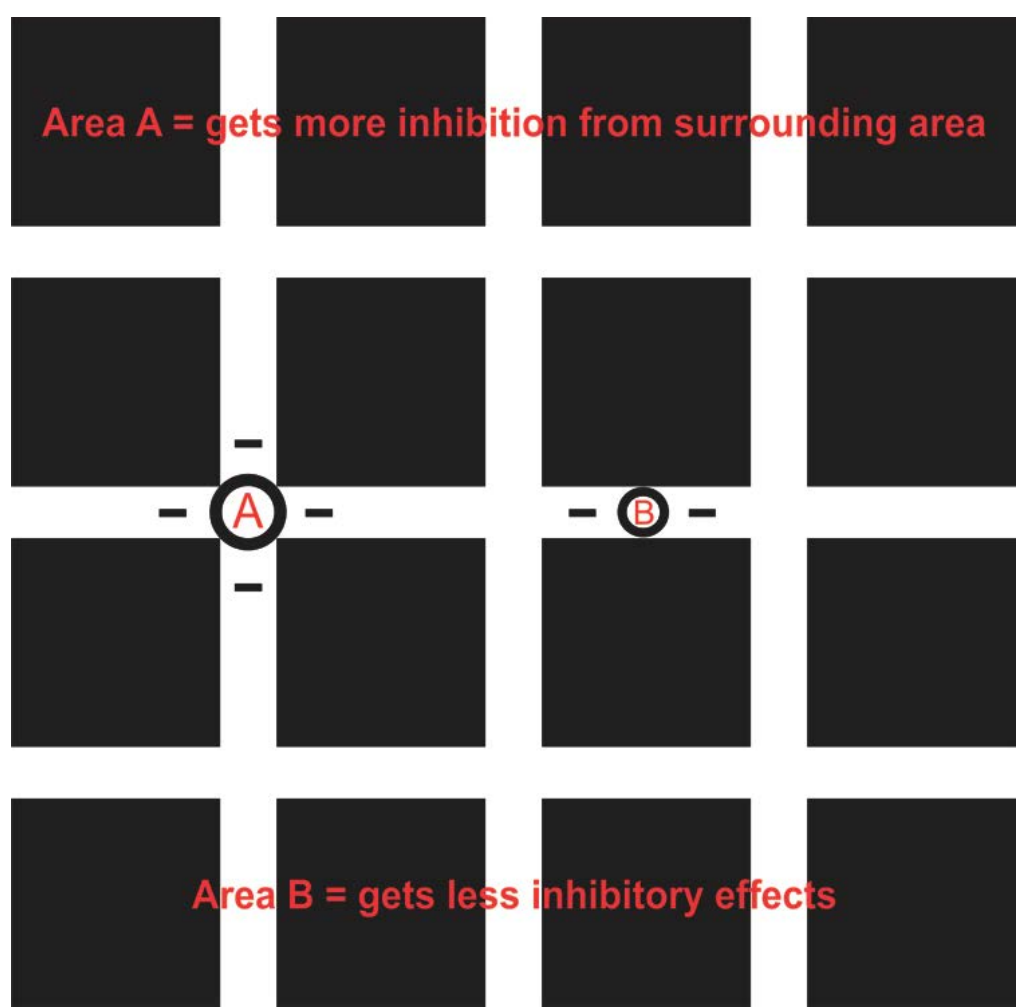

Figure 11. Traditional explanation of Hermann grid illusion.

S1 simple cells in the primary visual cortex (V1), whose receptive fields are elongated along their axis of orientation. According to them "illusory smudges are the result of the relative degree of activity of the on and off S1 cells at the intersections, as compared with activity at non-intersecting locations" [43]. But why one observes illusory spots in the middle of the intersections - their explanation leaves this question unanswered [42].

Optical mechanisms are inadequate overall to explain $\mathrm{H}$. Grid illusion, as the illusion disappears if the grid is tilted, or dis-shaped. According to this study, However, the exact reason for this illusion does not lie in any aberration of the optical mechanism; but the aberration lies in the process of translation of the optical stimuli to the visual perception in the brain. Because if the shape of the grid is altered, or the grid is tilted at 45 degree, the illusion disappears (Figure 12).

So what happens there?

We are very accustomed to grided views. Not only looking through the grided windows, but when we walk along the streets, blocks of houses, shops by the sides, with light or darkness coming through them, build our respective impression strong through the absorbed memories of grid views. Neglecting the curvature, the earth's surface is horizontal, and on it gravitational force is perpendicular. For that, our world is more horizontal and vertical in nature than any other form. And this is the reason behind Hermann-Grid optical illusion.

In Hermann-Grid illusion, we challenge 'the possibility' and 'the impossibility'. If the inside light is so bright to make the grid complete white, the darkness beyond the grid could not be as dark as complete black due to reflection and scattering of light by the grid. When we look at a bright light source, there appears a hallow around it due to scattering of light by the atmospheric molecules and particles. The darkness around the light source is smudged with same tinge of colour. To watch background as dark as complete black, the insight should be less lighted or the grid should be darkish. So the illusion appears at the peripheral vision which lies between the possibility and the impossibility. In Lingelbach illusion the borders have already been smudged with darkness. So the illusion does not appear at the borders as we are accustomed to see that type of darkness only through that colored grid.

In the vice versa case, if the background is bright white, bright yellow, bright red or of any bright colour, and the grid is of different colour, the illusion would also appear, because due to scattering of light, and to some extent reflection of the light from the inside objects including the observer, the grid will have a tinge of that colour.

So for theoretical purpose, for easier understanding, whether the background is dark or bright we can presume that background is scattering its own colour to produce its effect on the grid.

Now, why the darkness or smudge (effect) is more prominent at the intersections? - It is because of overlapping areas of scattering halos (from four quarters) at the intersections. Why we do not see the illusion when the grid is tilted at 45 degree or the shape of the grid is changed? Because we are not accustomed to see those views. We don't see anymore those as usual familiar views, we just see those 
as a design or a painting that does not challenge our memory.

To check this exposition right or wrong, the following thing was done. A black papergrid was put against the clear day light and photographed (Figure 13). As I said, there was white smudges at the intersections that dissipated along the borders also. But when I closely watched the photograph, I got that the white smudges are there, and it is not an illusion in reality. These are due to scattering of light by atmospheric molecules and particles over the dark areas. But here two more factors work - lateral inhibition and contrast colour perception. For that, margins of the grid-border appear relatively dark. So in an illusory view of the grid, the mind obviously imagines the peripheral view as it happens in reality as described previously (Figure 14).

Now we take, point $\mathrm{C}$ is the center of the grid junction, and is being illuminated by from the four quarters of scattered light. From a fixed distance, whether the $\mathrm{C}$ point will be the highest luminous point of the grid junction, that depends on two factors - (1) distribution of scattered light intensity and
(2) width of the grid bar.

Scattered light intensity depends on i) composition of the medium (air) - that is properties (size, shape, reflectivity) and density of the particles in the medium; ii) intensity and wavelength of the incident light; and iii) the angle of scattering. But the distribution of scattered intensity does not have a uniform value, as more light scattered and absorbed, less light enters into the successive layers. This light attenuation occurs in exponential fraction (Beer's law).

If the intensity of the incident light is $\mathrm{I}_{\mathrm{O}}$, then after travelling through the $\mathrm{M}$ distance of the medium, due to scattering attenuated light intensity $\mathrm{I}_{M}$ will be -

$$
\mathrm{I}_{\mathrm{M}}=\mathrm{I}_{\mathrm{O}} \times \mathrm{e}^{-\mathrm{TM}}
$$

$\mathrm{T}$ is the turbidity (dependent on particles' properties and concentration) of the medium.

For that, the chance of $\mathrm{C}$ point for being the highest luminous point of the grid junction increases when the width of the grid bar is less. This is true also for illusory case. But
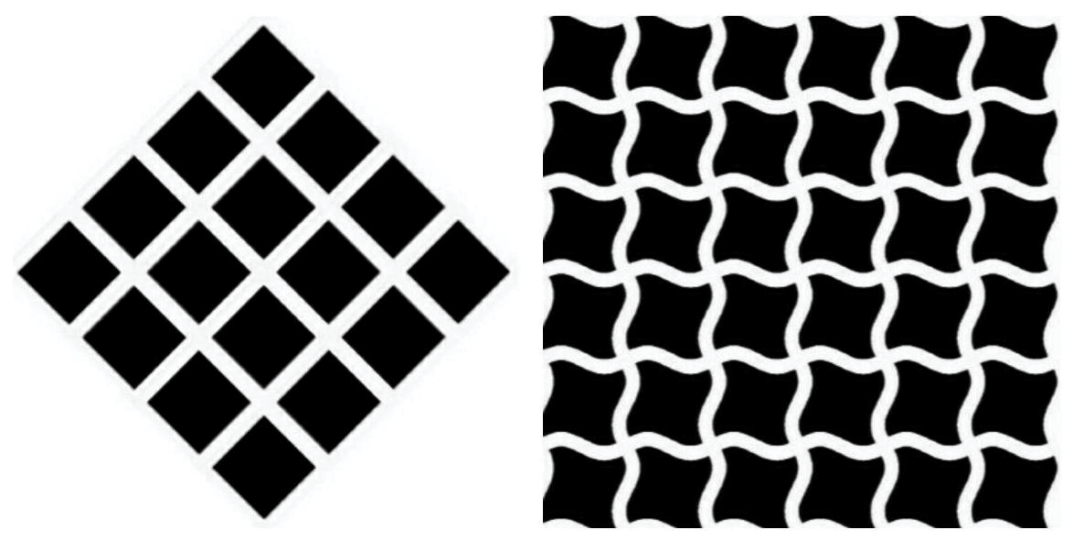

Figure 12. Tilted and Dis-shaped grid.
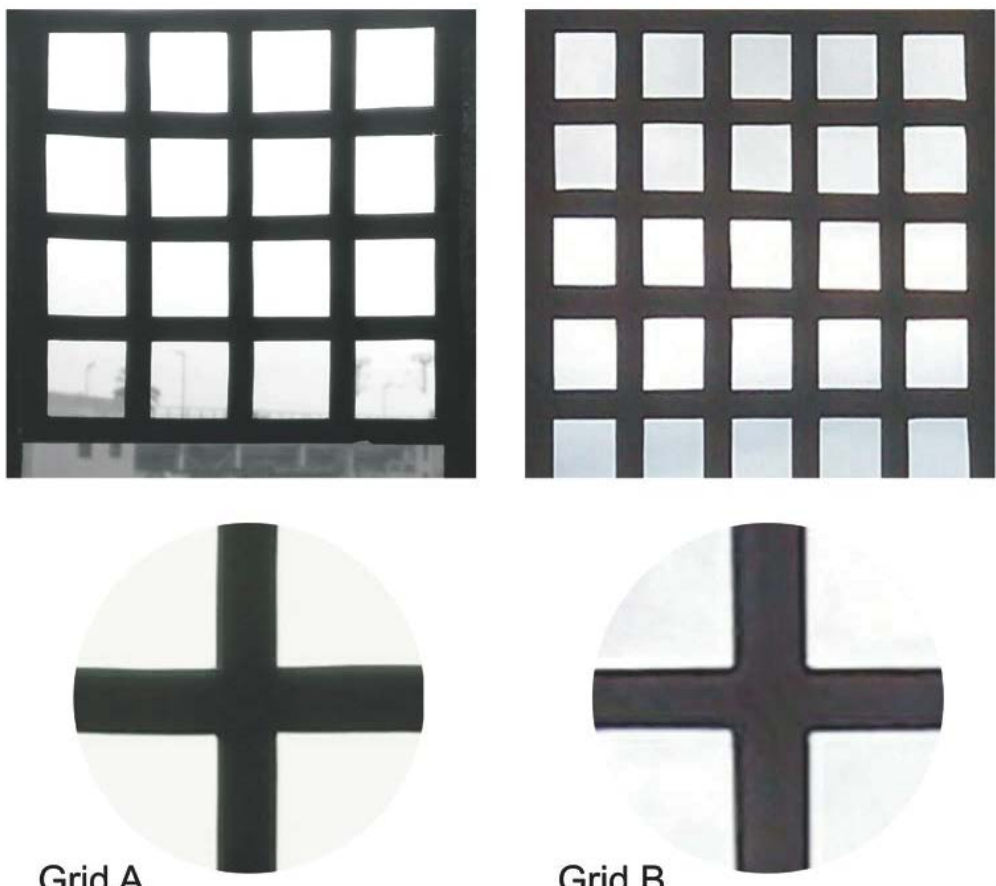

Grid A

Grid B

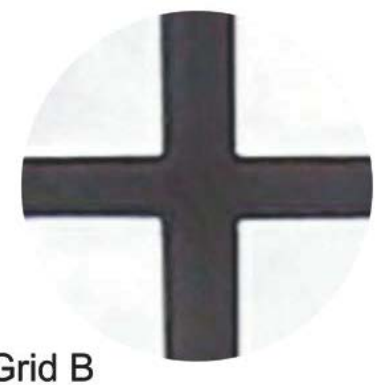

Figure 13. Grid in reality. 

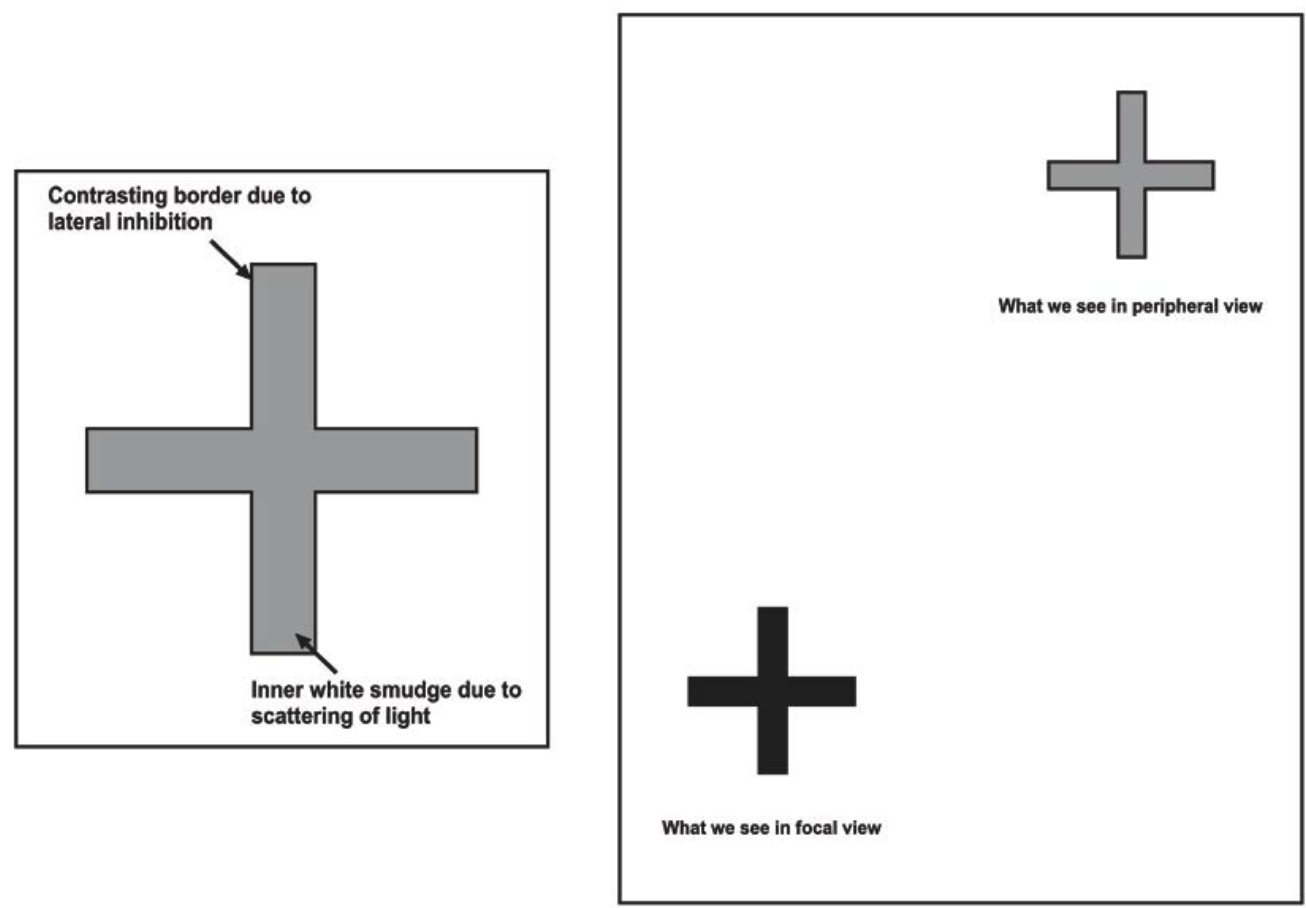

Figure 14. Explanation of H. Grid illusion according to present study.
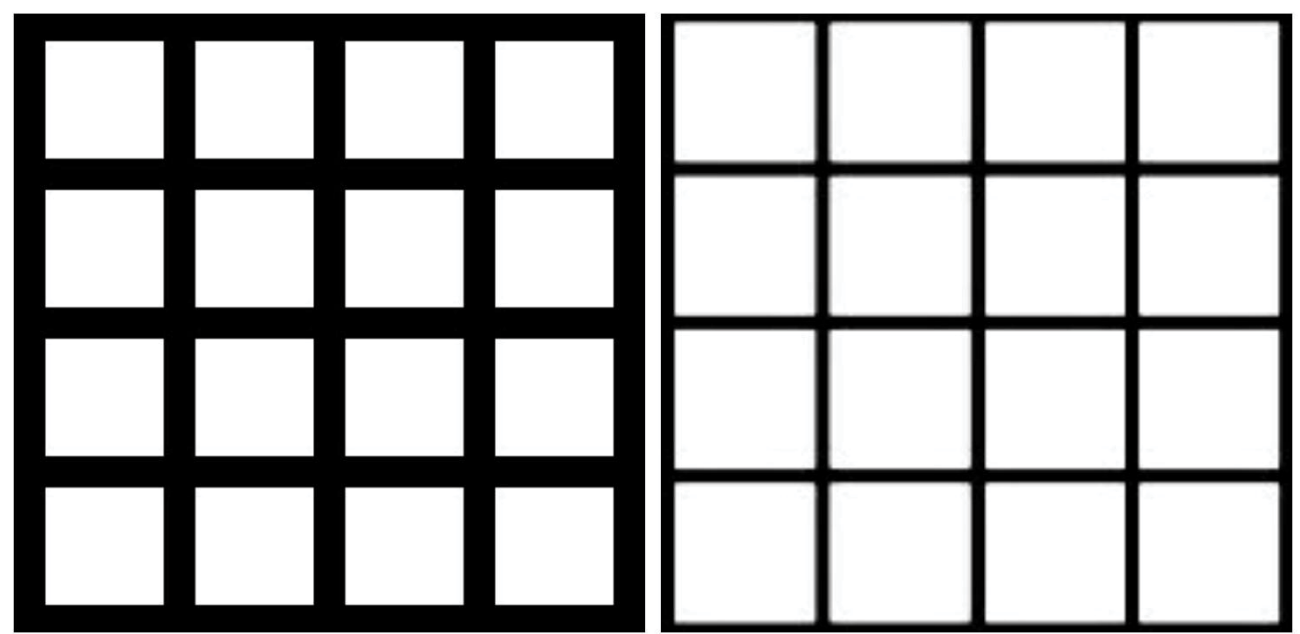

Figure 15. Here the illusory effect is more visible in grid b than grid a. And if we look at the images from more distance, the illusory effect will further grow.

to observe the effect, the grid width has to have a minimal value that depends on subjective visual acuity. Consider the images in Figure 15.

Here, the illusory effect is more visible in grid $b$, than grid a.

Now if the observer increases the distance from the grid, volume of the scattering medium will increase. But light attenuation will occur according to the inverse square law.

So, if the observer increases the distance from the grid, the effect (real and illusory) will increase, but it will be gradually blurred.

\section{Conclusion}

All this explanation with supportive presentations, states that the optical illusions are mostly dependent on our pre-

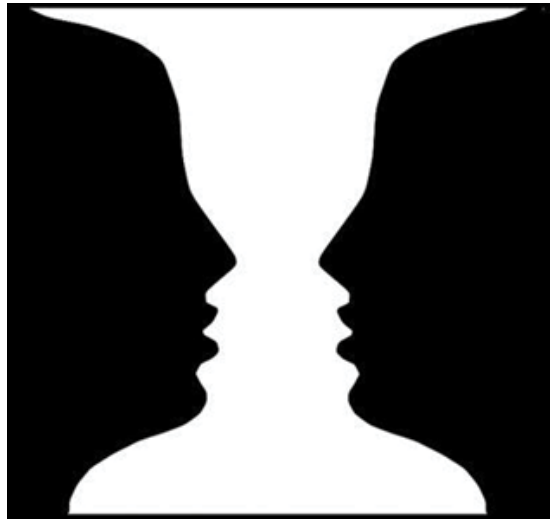

Figure 16. Rubin's vase.

embedded associated memories, regarding objects, views, situations, and perspectives. It includes visual memories, as well as other perceptual memories, auditory, olfactory, 
gustatory, tactile and proprioceptive memories. Helmholtz said, "every evening apparently before our eyes the sun goes down behind the stationary horizon, although we are well aware that the sun is fixed and the horizon moves" [7]. The reason behind it, is if the horizon moves we are supposed to get the perception of movement through our proprioceptive senses (vestibular apparatus, proprioceptive receptors in joints, muscles etc.). We are not accustomed to experience real movement without perception of it.

One questioned me, if most of the optical illusions are due to our cultivated associated memory, then if we empty our memories, there will not be any (optical) illusion. We cannot empty our memories on our own. But loss of memories (partial or complete) occurs in different psychological and medical cases, either temporarily or permanently. And yes, if associated memory is changed or lost, there will be difference in receiving optical illusions.

Our whole sets of associated memories make our knowledge. It not only includes memories as information, but our intellectual workings, emotional experiences are also assembled properly and stored as memories. All these acquisitions construct our integrated knowledge, experience, beliefs, ideas; and build the internal model of our mind. It is very obvious to say that it is largely influenced by family, culture, society and a person's surrounding world.

And our perception is greatly depended on this constituted knowledge and internal model of the mind. An ambiguous object interpreted differently by two different observers, exposes different structures or shapes of their subjective mind. Consider the following image (Figure 16).

One can interpret it reflexively as two faces, and another can interpret it as a flower vase. What will occur to a person's mind at the first sight of this image that depends on his pre-instituted knowledge, the whole organization of his associated and embedded memories. And it exposes one's internal shape of the mind, mental attitude, and way of looking to the outside world.

"Correspondence to:

Krishanu Kumar Das

HLG Hospital

Department of Medicine

Sen Raleigh Road

Asansol,

West Bengal 713304

INDIA

Tel: +917044728818; +918017377896

E-mail: dr.krisanudas@gmail.com

\section{References}

1. Brodbent DE. Perception and Communication. Pergamon Press. London. 1958.
2. Shiffrin RM, Schneider W. "Control and automatic human information processing: Perceptual learning, automatic attending, and a general theory". Psychological Review. 1977;84:127-190.

3. Yuille Alan, Kersten Daniel. "Vision as Bayesian inference: analysis by synthesis?", Trends in Cognitive Sciences, Special Issue: Probabilistic models of cognition. 2006;10(7):301-308.

4. Gibson JJ. The perception of the visual world. Houghton Mifflin Co. Boston. 1950.

5. Doorey M. James J Gibson: American Psychologist and Philosopher. Encyclopaedia Britannica. 2013.

6. Hochberg J. James Jerome Gibson: A biographical memoir. National Academy of Sciences. 1994.

7. Helmholtz $\mathrm{H}$ Von. Treatise on physiological optics. Southall J P C (Ed). Dover. New York.1910.

8. Klein GS. Perception, motives and personality. Knoff. New York.1970.

9. Witkin HA, Goodenough DR. Cognitive Styles : Essence and Origins. International Universities Press, New York. 1981.

10. Gibson EJ. Principles of Perceptual learning and development. Appleton-Century-Crofts. New York. 1969.

11. Arnheim R. Visual Thinking. University of California Press. Los Angeles. 1997.

12. Pinker S. Visual Cognition: Computational Models of Cognition and Perception. Cambridge MA: MIT Press. 1986.

13. Pinker S. How the Mind Works. W. W. Norton \& Company. New York.1997.

14. Pinker S. Visual Cognition: Computational Models of Cognition and Perception. Cambridge MA: MIT Press. 1986.

15. Sacks O. The Mind's Eye. Vintage Books. New York. 2010.

16. Grimaldi Madalena. Illusions: The magic eye of perception. Transtechnology research paper. Plymouth University. UK. 2013.

17. Kanizsa G. "Subjective Contours". Scientific American. 1976;234(4):48-52

18. Navon D. Forest before trees: The precedence of global features in visual perception. Cognitive Psychology. 1977;9:353-83

19. Cassia VM. Dominance of global visual properties at birth. J of Experimental Psychology. General. 2002;13(3):398411.

20. Gregory RL. Eye and Brain : The psychology of seeing. McGraw-Hill. New York. 1978. 
21. Coren S, Girgus JS. Seeing is deceiving : The psychology of visual illusions. Erlbaum. Hillsdale. 1978.

22. Gibson JJ. The ecological approach to visual perception. Houghton Mifflin. New York. 1979.

23. Biederman I (1987). "Recognition-by-components : A theory of human image understanding". Psychological Review. 1987;94:115-47.

24. Biederman I. "Geon theory as an account of shape recognition in mind and brain. Irish $\mathrm{J}$ of Psychology. 1993;14(3):314-27

25. Morgan CT, King RA, Weisz JR, Schopler J. Introduction to Psychology. McGraw Hill Education (India) Private Limited. 1993.

26. Ernst Mach. Psychology and the life sciences : Mach Bands". In Stanford Encyclopedia of Philosophy. 2009.

27. Jamieson RK, Crump MJC, Hannah SD. "An instance theory of associative learning." Learning and Behavior. 2012;40:61-82.

28. Suzuki W A. Associative learning and the Hippocampus. Psychological Science Agenda. American Psychological Association. 2005.

29. Mackintosh NJ. "An analysis of overshadowing and blocking." Quarterly Journal of Experimental Psychology. 1971;23:118-25.

30. Mackintosh NJ. "A theory of attention : Variations in the associability of stimuli with reinforcement." Psychological Review. 1975;82:276-298.

31. Rescorla RA, Wagner AR. "A theory of Pavlovian conditioning : Variations in the effectiveness of reinforcement and nonreinforcement." Classical conditioning II : Current theory and research. AppletonCentury-Crofts, New York. 1972;64-99.

32. Chapman GB, Robbins SJ. "Cue interaction in human contingency judgment". Memory and cognition. 1990;18:537-45.

33. Dickinson A, Shanks D. Animal conditioning and human causality judgement." Perspectives on learning and memory, L. G. Nilsson \& T. Archer (Eds.), Hillsdale: Erlbaum. 1985;167-91.

34. Miller RR, Matute $H$. "Biological significance in forward and backward blocking : Resolution of a discrepancy between animal conditioning and human causal judgement." Journal of Experimental Psychology: General. 1996;125:370-86.

35. Van Hamme LJ, Wasserman EA (1994). "Cue competition in causality judgements: The role of nonpresentation of compound stimulus elements." Learning and Motivation. 1994;25:127-51.

36. Ward WD, Jenkins HM. "The display of information and the judgement of contingency". Canadian Journal of Psychology. 1965;19:231-41

37. Shaklee H, Tucker D. "A rule analysis of judgements of covariation between events." Memory and Cognition. 1980;8:208-24.

38. Waldmann MR, Holyoak KJ. "Predictive and diagnostic learning within causal models : Asymmetries in cue competition". Journal of Experimental Psychology: General. 1992;121(2):222-36.

39. Catena A, Perales JC, Maldonado A. "Judgement frequency effects in generative and preventive causal learning". Psycologica. 2004;25:67-85.

40. David SS. "Peripheral vision and child pedestrian accidents". British Journal of Psychology. 1986;77(4):43350 .

41. Baumgartner G. "Indirekte Grobenbestimmung der rezeptiven Felder der Retina beim Menschen mittels der Hermannschen Gittertauschung" Pflugers Archiv fur die gesamte Psychologie. 1960.

42. Geier J. "Straightness as the main factor of the Hermann grid illusion". Perception. 2008;37(5):651-5.

43. Schiller PH, Carvey CE. "The Hermann grid illusion revisited". Perception.2005;34:1375-97.

\section{*Correspondence to:}

Krishanu Kumar

DasHLG Hospital

Department of Medicine

Sen Raleigh Road

Asansol

West Bengal 713304

India

Tel: +917044728818; +918017377896

E-mail: dr.krisanudas@gmail.com 